\title{
Binocular fusion and contour suppression
}

\section{ALVIN G. GOLDSTEIN ${ }^{1}$ UNVERSITY OF MISSOURI}

\begin{abstract}
Data from three separate investigations are presented to support the conclusion that suppression of visual contours occurs during binocular fusion of identical figures. These data are discussed in relation to examples of confirming and disconfirming results of other investigators.
\end{abstract}

Although we view our world through two eyes, and each eye initiates neural excitation, under normal conditions we rarely see double images. Attempts to understand and to explain single vision have been remarkably persistent, as attested to by the fact that the problem was recognized at least two hundred years ago, and remarkably insoluble, as proven by the recent renewed interest in the same problem. The present report describes another attempt to solve that same problem.

Two fundamental ideas may be distilled from the various theories offered to explain the fate of the "second" image. The concept of fusion has been invoked, where unification is achieved by assuming that both the left and right eyes' excitations are represented in the cortical projection area and are fused in some unexplained manner into a single percept. The rationale for maintaining that both excitations are functional in perception probably can be traced to the stereopsis theory of depth, where two slightly different "half-images" are needed to explain perceived depth.

Single vision is explained in another theoretical view by assuming that only one image reaches the projection area, i.e., in normal binocular vision the "second" image is suppressed. This viewpoint skirts the powerful theory of stereopsis by offering evidence to show that phenomenal depth is not destroyed during binocular rivalry of two stereo half-images, implying, of course, that in normal binocular vision, both excitations are not required for depth perception (Asher, 1953; Verhoeff, 1935).

It could be argued that the very existence of binocular rivalry (BR) supports the thesis that true fusion does not occur in normal binocular vision. This conclusion has some merit only if it is assumed that BR-or some form of suppression-is not an artifact, but reflects the normal function of the visual system. Thus, we do not normally see or report alternations (BR) in our perceptions of single real objects because we are not able to distinguish the suppressions of each almost-identical half-image since they are perfectly overlapping, i.e., fused. In general, demonstrations of the occurrence of BR in normal, binocular vision employing phenomenal observations have not been convincing(Washburn \& Manning, 1934).

In reanalyzing data from a study of BR rates as a function of duration of observation time (Cogan \& Goldstein, 1967), evidence was uncovered suggesting that perceptual alternations of the rivalry stimuli were less frequent during the initial few seconds than during the subsequent periods of observation. Further analysis indicated that simultaneous perception of left and right eye stimuli were more frequently reported during the initial $5 \mathrm{sec}$ of a BR trial than in any subsequent 10-sec period. We reasoned that these data could be understood if it is assumed that BR does not occur during the period of normal binocular vision immediately preceding the experimental conditions. Therefore, when the visual system is confronted during the experiment with differing stimuli impinging on corresponding retinal points in each eye, BR may take some time to "get started."We speculated further that if a rivalry response was less frequently produced to the rivalry-eliciting stimulus in the initial seconds of viewing, then perhaps $B R$ is not a normal function of the visual system, but is an induced phenomenon, occurring only in the presence of nonfusing half-images. As will become evident in a moment, these inferences led to in teresting empirical results that do not seem to point in this direction.

At the time we were speculating about these data, Fox and McIntyre (1967) published evidence in support of a suppression theory of binocular vision. If I understood their report, they first manipulated their stimulus conditions for their three Ss and obtained a .63 mean probability of correct identification of a probe stimulus (one of three letters of the alphabet) introduced to either eye during fusion of two white, uncontoured squares (for details, see report by Fox and McIntyre, 1967). With this baseline probability established, they then demonstrated suppression when one of the uncontoured squares (simple target) was "fused" with a multicontoured square (complex target) and the probe was introduced into the eye viewing the simple target. Under these conditions, mean probability of correct identification was .49 . When the probe was introduced into the eye viewing the complex target in the complex-simple condition, probability of correct identification was increased reliably over the baseline or simple-simple condition (mean probability = .73). Primarily on the basis of the obtained difference between the two simple-complex conditions and the complex-complex condition, the authors concluded that competitive interocular suppression was produced by the presence of complex targets in each eye (p. 144). The fact that response accuracy in the simple-simple (mean $=.63$ ) and complex-complex (mean = .63) conditions were not significantly different makes this general conclusion less convincing. It is of some importance that the latter two conditions are the only "true" fusion (that is, nonrivalry) conditions in the experiment, and they differ mainly on amount of contour stimulating each eye, a variable that should affect the amount of suppression generated. The remaining two simple-complex conditions essentially are examples of total visual dominance caused by grossly unequal visual input to one eye with little or no input to the other eye. Dominance of this sort does not as a rule change to phenomenal rivalry, but it is a "rivalry type" of visual input.

Since Fox and Mclntyre's (1967) report represented one of the clearest attempts to solve the problem of binocular fusion, replication was warranted for at least two reasons: The solution of the problem is critically important for our understanding of the visual mechanism, and there is perhaps some reason to question the general conclusion of the experiment.

In the remaining sections of this report, we will first describe a simple method for determining whether or not suppression exists during binocular fusion of identical shapes, i.e., during normal binocular vision. Included with this description will be the results of using that method with several Ss. Subsequently, some data from a more refined method will be offered in support of the findings of the first study. Finally, the conclusions will be discussed with regard to recent evidence presented in two other investigations on closely related visual problems.

\section{EXPERIMENT 1}

Method

Generally speaking, we have attempted to demonstrate the perceptual effect of suppression of one eye's input immediately 


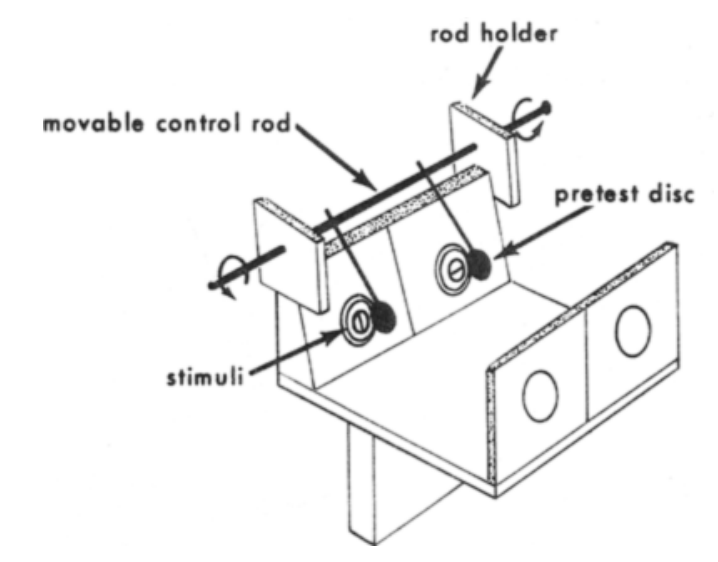

SCHEMATIC OF MODIFIED KEYSTONE TELEBINOCULAR

following conditions of binocular fusion. More precisely, by using a stereoscope, Ss were confronted with two identical pretest stimuli, one to each eye, an arrangement that always produced a single percept indistinguishable (phenomenally) from a normal binocular percept. After a short observation period, the pretest fusion-inducing stimuli were quickly removed, thereby uncovering two nonidentical test targets, a horizontal line for one eye and a vertical line for the other, arranged so that they were congruent with the former positions of the pretest stimuli. If BR was "going on" during the preceding single vision (fusion) condition, then it was reasonable to expect that $\mathrm{Ss}^{\prime}$ immediate perception of the test stimulus would be fragmentary, indicating that some part of the test target was not visible. That is, suppression from the pretest "fused" condition would influence or carry over into the test condition. On the other hand, if BR was absent during the fusion condition, we would expect the immediate perception of the test stimulus to be veridical, to lack fragmentation. That is, the images from each eye would be intact, and no evidence of suppression would be found.

At first, a fairly crude experiment was devised to test these expectations. A Keystone Telebinocular was modified as shown in Fig. 1. Referring to Fig. 1, two identical, dull black discs (pretest discs) are each rigidly welded to the two vertical rods, which are in turn rigidly connected to the longer control rod. Two accurately drilled holes in the plastic blocks (rod holders) serve as bearings to receive the control rod, permitting it to rotate along its axis without binding. Rapid clockwise rotation of the assembly moves both black discs through a 180-deg arc, removes the pretest fusion stimuli, and uncovers the test stimuli beneath the discs. All stimuli were placed at the far-point position of the instrument. Each disc was slightly dome-shaped, and subtended $4 \mathrm{deg}$ of visual angle. Two sets of black-on-white test stimuli were used. The only important difference between the stimuli was the visual extent of the vertical and horizontal lines; in the large stimulus, the lines subtended $3.5 \mathrm{deg}$, whereas in the small stimulus pair, the lines subtended 1 deg of visual angle (Fig. 2).

When $S$ looked into the apparatus, he saw a single black disc attached to a single vertical rod. The discs always completely covered the vertical and horizontal lines of the test stimuli until the discs were lifted. Single vision was never disrupted in these conditions. Removal of the disc by $\mathrm{E}$ was "instantaneous," without noticeable movement of the discs. Illumination was provided by a $40-W$ bulb.

Subjects were summer-session students (17 to 26 years old) enrolled in General Psychology at the University of Missouri. Of the total $40 \mathrm{Ss}$, only 12 wore some kind of corrective lenses. Fairly stringent criteria were used to screen $\mathrm{Ss}$ on measures of acuity, phoria, and depth perception. In addition to (Keystone) phoria screening tests, all Ss had to "hold" a monocular cross within a monocular circle without benefit of fusion stimuli for at least $30 \mathrm{sec}$, a task that requires superior eye muscle balance. Failure to meet any one of these criteria immediately eliminated the prospective $S$. In addition, all Ss had to demonstrate during the experimental session (see below) normal ability to report BR under appropriate stimulus conditions.

Although a two-group design was employed, the details of which will be given below, procedures for obtaining the dependent measure were the same for all Ss. Thus, every $S$ was required to fixate the center of the singly perceived black disc for a period of a few seconds. After giving a "ready" signal, E rapidly rotated the discs upward, at which time $S$ reported whether or not the horizontal and vertical lines in the test stimulus (Fig. 2) were completely in tact
Fig. 1. Schematic drawing of Keystone Telebinocular showing the modifications used in Experiments 1 and 2.

at the instant these lines came into view. If both lines were intact, $S$ responded with "both," whereas if any part of one line was missing or parts of both lines were missing, $S$ responded by saying "one." "Both" and "one" responses were recorded by E. Two levels of instruction and familiarization with BR were used. One group, comprising half the Ss, remained essentially naive regarding BR; these Ss were given only one training trial immediately before data collecting trials were initiated. Ss in this group, referred to hereafter as Untrained, were given their vision tests, instructions, and experimental trials in a single experimental session at the end of which BR rates were obtained on each of the two test stimuli, during $1 \mathrm{~min}$ of uninterrupted viewing.

A second group of $20 \mathrm{Ss}$, hereafter referred to as Trained, was required to participate in two sessions separated by $24 \mathrm{~h}$. During the first session, these Ss experienced BR for several minutes, followed by several training trials in which the discs uncovered the test stimuli. During these trials $S$ was asked to report on the perceived state of the test stimuli but no record of responses was taken in this familiarization session. Measures of BR rates during $1 \mathrm{~min}$ of uninterrupted observation were recorded for each test stimulus at the end of this first session. Upon returning for the second session, data collection on the experimental task immediately followed $\mathrm{E}$ 's recitation of the same instructions given to all Ss in the experiment. ${ }^{2}$ All Ss responded on 15 useable trials per test stimulus. A trial was not used if $S$ reported that he blinked at a critical period, or if the test stimuli were seen double, i.e., fusion was lost on the experimental trial. Both of these events were rarely encountered.

\section{Results and Discussion}

As explained earlier, two responses were tabulated. The response "both" indicated that the test stimuli were seen to be completely intact at the moment they were uncovered, suggesting the absence of suppression, i.e., lack of BR. The response "one" indicated perception of fragmentation of the test stimuli, suggesting the presence of suppression.

Per cent "both" responses have been entered into Table 1, with separate values shown for the Trained and Untrained groups' responses to the $1-\mathrm{deg}$ and $3.5-\mathrm{deg}$ test stimuli. It is obvious that nonsuppression responses to the test stimuli comprised the most common finding in all conditions, but in no condition did this 
Table 1

Mean Per Cent Nonsuppression Responses

Stimulus Size

\begin{tabular}{|c|c|c|c|}
\hline & & & \multirow[b]{2}{*}{ Total } \\
\hline & $1 \mathrm{deg}$ & $3.5 \mathrm{deg}$ & \\
\hline $\begin{array}{l}\text { Trained } \\
\text { Untrained }\end{array}$ & $\begin{array}{l}70 \\
83\end{array}$ & $\begin{array}{l}64 \\
71\end{array}$ & $\begin{array}{l}67 \\
77\end{array}$ \\
\hline Total & 77 & 68 & 72 \\
\hline
\end{tabular}

mean value approach $100 \%$. Although Trained Ss reported fewer fusion responses than Untrained Ss to both test stimuli, this difference was not reliable in either case, suggesting that the task was simple enough for naive $S s$ to perform adequately. Test stimulus size was a reliable variable for the Untrained Ss, with the larger stimulus eliciting 9\% more nonsuppression responses, but not for the Trained Ss. This suggests that the training may have improved Ss' ability to pay attention to a larger visual field, and report occurrences of missing contours.

Individual differences were unusual in their persistence. A few Ss report suppression on all or almost all trials, some never report anything but completely intact lines, and many report suppression-like responses on some trials but not on others. In this connection it is important to remember that all Ss were able to experience binocular rivalry.

Responses indicating nonsuppression ("both") comprised $72 \%$ of the total. These responses are interpreted to demonstrate that on a large number of trials, suppression is not a component of fusion. The remaining $28 \%$ of the responses indicated that during these trials some suppression generated during binocular fusion "carried over" into the nonfusion test condition. Conventionally, in an experiment with only two possible responses, the smaller, nonsignificant proportion of responses to the experimental stimulus would be considered to be "errors" of one kind or another. But here; the response "both" is assumed to have an expectancy value of $100 \%$. We believe this to be true because on every trial, the horizontal and vertical test lines were always there, unbroken and complete. Exposure time and intensity of illumination were not manipulated and were in fact more than adequate for the task of determining the perceptual condition of the two test lines. In essence, the perceptual task confronting the $\mathrm{S}$ was ridiculously simple. With one exception, to be taken up in a moment, there is no reason to expect $S$ to misperceive and report an incomplete or totally missing line unless suppression was occurring.

There may be one exception to this expectation of errorless reporting. Perhaps $S$ could not report what he saw at the instant the test stimuli were uncovered, but delayed a fraction of a second and reported the subsequent $B R$ in response to the test stimuli. This interpretation is weakened by the fact that the Trained group made $10 \%$ fewer suppression responses (but not significantly so) than the Untrained Ss. If suppression responses were "errors," it would be plausible to have expected the training to significantly reduce their frequency, but this was not the case.

\section{EXPERIMENT 2}

Replication of these data were obtained in another experiment using the same apparatus. Three highly trained Ss viewed the pretest black discs in addition to four new pretest stimuli differing roughly along the contour-density continuum. 3 Suppression responses following the pretest black disc came to $29 \%$, a value almost identical to the finding of the original study. Suppression responses to all five pretest stimuli came to $28 \%$ (of 225 total responses). No systematic differences in response frequency to the various contour densities were evident. ${ }^{4}$ Once again, large individual differences were encountered, and this in spite of Ss' planned familiarity with the task and the E's added cautionary instructions regarding the fact that $S$ "... may see the same thing over and over again, but you are to report exactly what you see."

If these results are reliable, and the assumptions underlying the experimental manipulations are tenable, it appears that even a fairly crude-perhaps inelegant-method is capable of
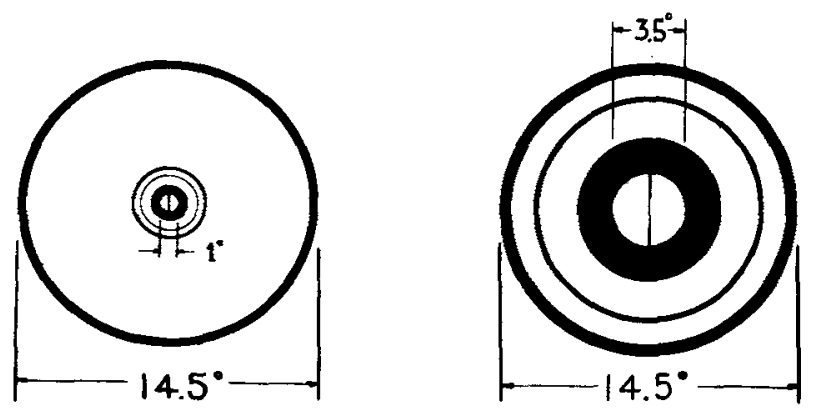

demonstrating suppression during binocular fusion. Obviously the method is not without its faults, although its simplicity may outweigh some of its drawbacks. Nevertheless, in an attempt to verify these findings, another investigation was designed, incorporating essentially the same strategy but using the more sophisticated switching circuitry of a tachistoscope instead of the Keystone stereoscope. Inasmuch as this method and the results will be discussed in detail in a subsequent report, only the essentials will be offered here.

\section{EXPERIMENT 3}

The binocular eyepiece of a Scientific Prototype two-channel tachistoscope (Model 800-E) was masked except for two 13-mm-diam circular openings, separated by $60 \mathrm{~mm}$ on center. This modification made it possible to stimulate Ss' left and right eyes independently. All stimuli were situated $81 \mathrm{~cm}$ from Ss' eyes. When left and right eye pretest stimuli were mounted in the fixation field of the instrument, a single fused image was seen. Similarly, two test stimuli mounted in the stimulus field of the instrument, exactly congruent with those in the fixation field, also were seen as single images. Essentially, with these modifications, the tachistoscope was used as a stereoscope with alternating stereo fields; the electronic switching circuits are analogous to the mechanical method of uncovering the test stimuli in the previous study.

In this experiment, every stimulus was composed of two black-on-white concentric circles, subtending $1 \mathrm{deg}$ of visual angle. For test stimuli, the now familiar horizontal and vertical lines were inscribed within the smaller circle. In the case of pretest stimuli, various line patterns were inscribed within the boundaries of the inner circle. For example, one set of stimuli was composed of cross-hatched lines. Obviously, these pretest patterns were identical for both eyes, and were analogous to the black discs in the original study in that fusion readily occurred. Pretest viewing time was $3 \mathrm{sec}$, followed by $500 \mathrm{msec}$ exposure to the test stimuli with 0 sec delay between removal of pretest stimuli and presentation of test stimuli. The test stimuli were followed by complete darkness. Although Ss were not actually trained by exposure to the experimental stimuli, they were given general practice on making responses to

Fig. 2. Visual angles subtended by "small" (on left) and "large" test stimuli in Experiments 1 and 2. Each diagram represents one-half of the stereoscopically presented stimulus. The pretest stimuli (black discs) measured $4 \mathrm{deg}$ and were exactly concentric with the test stimuli. 
perceptual stimuli in the tachistoscope, including practice in fusing stimuli seen through the two small openings in the eyepiece mask.

Instructions, general procedure, and S's response were all similar to analogous details of the earlier study with one major exception. On predetermined trials, the test stimulus was replaced with a "catch" stimulus designed to measure S's ability to report the presence of a $2-\mathrm{min}$ visual angle gap in the vertical or horizontal test stimulus line. The gap stimulus was identical to the test stimulus in all other respects. Correct response to the small break, of course, indicated that $S$ was paying attention to the stimulus, and could discriminate a small missing contour. More importantly, if $S$ reported the presence of the gap, and on other trials also reported that some part of the intact test stimuli were occasionally missing or incomplete, then it would be convincing evidence for the suppression interpretation already of fered.

The results strongly support the findings of the original investigation. This conclusion is true when using only the data from those Ss who make no error in reporting the gap in the catch stimuli. These $S$ s still report parts of the test stimulus to be missing when, in fact, the lines are completely intact. Thus, using a procedure that controls the duration of pretest inspection, switches from pretest stimuli to test stimuli with zero time delay, and presents the test stimulus for a very short (500 msec) duration, the conclusion reached is essentially unchanged from that implied by the data of the first study and its replication. During binocular fusion, suppression can be demonstrated on some, but not all, trials. This suggests that suppression during normal binocular vision may not be an all or none phenomenon. These conclusions are in agreement with the conclusions offered by Fox and McIntyre (1967).

Perhaps these conclusions may be understood in the context of the model one employs for describing $B R$. If $B R$ is conceived of as altemating total suppressions, i.e., only one eye's input is perceived at any moment in time, then the $72 \%$ nonrivalry responses represent evidence for nonsuppression during single vision, and the $28 \%$ rivalry-type responses represent evidence of suppression. Thus, in the phenomenon called single vision, two modes of functioning are implicated, with the implied question, why should there be two modes? On the other hand, if the BR model allows for alternating dominance of each eye's input (at least for targets of very small visual angle) with periods of time where neither eye is suppressed but both inputs are "dominant"-both are contributing equally to the momentary perception-then the data may be understood as follows. If a response is sampled from this system (using the procedure of this experiment) at the point in the cycle where input from neither the right nor the left is dominant, a large number of responses would be tabulated as "both" and a smaller number of responses would be tabulated as "one," demonstrating that rivalry is indeed a component of single vision. There would be no need here to postulate two modes of perceptual function.

\section{DISCUSSION}

Using somewhat different experimental manipulations, Fox and McIntyre (1967) and the present author have arrived at similar theoretical positions with regard to one aspect of binocular fusion. That is, some of the time the two eyes operate independently. Strictly speaking, this conclusion should be limited to the fovea or to an area immediately adjacent to the fovea. Thus, it is important to consider an experiment, employing a method different from the two already described, in which the results appear to lead to opposing conclusions. Greenspon and Eriksen attempted to assess the extent of binocular interaction by presenting "...two dissimilar letters simultaneously or at a short delay interval of $0-200 \mathrm{msec} \ldots$ on corresponding or noncorresponding retinal areas [1968, p. 93]." In all conditions, a binocular " $x$ " was fixated by $S$ before the target stimulus was presented, a procedure that is analogous to the pretest stimuli in the present investigation. They concluded that the two eyes do not operate independently when stimulation falls upon corresponding retinal points. If two different stimuli are presented on corresponding areas of the two eyes within somewhat less than $100 \mathrm{msec}$ of each other, the identification of both stimuli is impaired (p. 95). Simultaneous presentation caused maximum reduction in identification, a finding not at all in agreement with the notion that suppression is a component of binocular fusion. Obviously, this disagreement cannot be settled here, and there may be enough differences in details of procedure and method to make the comparison of somewhat doubtful usefulness. But there is little question that the present author's investigation, and Fox and McIntyre's (1967) and Greenspon and Eriksen's (1968) were all interested in determining the fate of simultaneous binocular visual inputs, a similarity in purpose that requires that differences in conclusion be treated as a serious matter.

Also relevant to the question at issue, is a recent report of research on the "Köllner effect." Crovitz and Lipscomb (1963) simultaneously presented fairly large (13 deg) uncontoured chromatic fields, one to each eye for exposure duration of $100 \mathrm{msec}$. Perceptual dominance of the nasal hemiretinae was demonstrated in that $50 \%$ of the percepts reported by the Ss involved the colors seen in the temporal visual fields of each eye, and only $9 \%$ could be scored as rivalry (that is, one-eye responses). These data of fer little comfort to a suppression theory of fusion, nor do they appear to be in agreement with the findings of the present experiment. Another aspect of the investigation was more encouraging. Without modifying the duration of exposure or the design of the experiment, Crovitz and Lipscomb also presented two hues to each eye, vertically split (e.g., left eye-left half green, right half red; right eye-left half red, right half green). Again the most common report (approximately 36\%) corresponded to the stimulus activating the nasal retinae of each eye, but now the next most common response (32\%) corresponded to the stimuli activating the temporal and nasal hemiretinae of one eye, a clear indication of binocular rivalry, or suppression of the other eye's visual input. Apparently the introduction of a contour-the demarcation between the two hues in each eye-increased the probability of suppression. These data are in keeping with the conclusion that binocular vision appears to involve suppression of one input, and furthermore, that the presence of visual contours enhances suppression. Perhaps this is additional evidence for believing that binocular rivalry between hues is substantially different from binocular rivalry between contours.

In the way of summary, then, all three experiments are supportive of the conclusion that at least some of the time during binocular fusion of identical contours, a measurable amount of suppression can be detected. These experiments were designed to determine whether or not suppression occurred as a consequence of fusion, but were not designed to either measure the relative amount of suppression as a function of the kind of antecedent conditions of stimulation, or to determine the nature of these antecedent conditions. Some of these questions are being investigated at this time.

\section{REFERENCES}

ASHER, H. Suppression theory of binocular vision. British Journal of Ophthalmology, 1953, 37, 37-49.

COGAN, R., \& GOLDSTEIN, A. G. The stability of binocular rivalry during spaced and massed viewing. Perception \& Psychophysics, 1967, 2, 171-174.

CROVITZ, H. F., \& LIPSCOMB, D. B. Dominance of the temporal visual fields at a short duration of stimulation. American Journal of Psychology, 1963, 76 631-637.

FOX, R., \& McINTYRE, C. Suppression during binocular fusion of complex targets. Psychonomic Science, 1967, 8, 143-144. 
GREENSPON, T. S., \& ERIKSEN, C. W. Interocular non-independence. Perception \& Psychophysics, 1968, 3, 93-96.

VERHOEFF, F. H. A new theory of binoculas vision. Archives of Ophthalmology, 1935, 13, 151-175.

WASHBURN, M. F., \& MANNING, P. Retinal rivalry in free vision of a solid object. American Journal of Psychology, 1934, 46, 632-633.

\section{NOTES}

1. Address: McAlester Hall, University of Missouri, Columbia, Missouri 65201.

2. The following instructions were read to all Ss. "When you look into this instrument you will see a dull black disc. I want you to look at the center of the disc. While you are looking at the center, I will very quickly remove the disc and uncover a figure under the disc. The instant the figure appears, tell me what the figure is. (At this point, E demonstrates one trial to S.) Now, on the following trials always fixate the center of the disc. I will give you the ready signal and very shortly after the ready signal, the disc will be replaced with a figure. Your task is to pay close attention to the figure you see the instant the disc is removed If the very first thing you see is a vertical and horizontal line then say, "both'; if you see a vertical line alone, or a horizontal line alone, then say, 'one.' You also should report 'one' if any of the two lines has some part missing. Let me impress you with the importance of reporting what you see the instant after the disc is removed. This is not easy to do because what you will see will rapidly change. Once again, report 'both' if you see both lines intact. Report 'one' if either line is partially or wholly missing."

3. The pretest stimuli, all inscribed within a crick subtending $4 \mathrm{deg}$ of visual angle, are described as follows: 16 vertical lines; a spiral pattern with several intersecting lines; the black disc; a grid work of 20 vertical and 10 horizontal white lines on a dark grey background; 4 vertical black lines, each approximately $.5 \mathrm{deg}$ in thickness.

4. This is not meant to imply that frequency of suppression responses was not a function of the various pretest stimuli. Suppression responses did vary, but at this time no clear independent variable can be identified.

(Accepted for publication May 5, 1969.) 